\title{
Reflection-based fibre-optic refractive index sensor using surface plasmon resonance
}

\section{P. Hlubina \\ petr.hlubina@vsb.cz \\ M. Kadulova \\ D. Ciprian}

\section{J. Sobota}

\author{
Department of Physics, Technical University Ostrava, 17. listopadu 15, 70833 Ostrava-Poruba, Czech \\ Republic \\ Department of Physics, Technical University Ostrava, 17. listopadu 15, 70833 Ostrava-Poruba, Czech \\ Republic \\ Department of Physics, Technical University Ostrava, 17. listopadu 15, 70833 Ostrava-Poruba, Czech \\ Republic \\ Institute of Scientific Instruments of the ASCR, v.v.i. Kralovopolska 147, 61264 Brno, Czech Republic
}

\begin{abstract}
A reflection-based fibre-optic refractive index sensor using surface plasmon resonance (SPR) in a thin metal film sputtered on a bare core of a multimode optical fibre is presented. The sensing element of the SPR fibre-optic sensor is the core of a step-index optical fibre made of fused silica with a gold film double-sided sputtered on the whole core surface, including the core end face. Consequently, a terminated reflection-based sensing scheme to measure the refractive indices of liquids is realized. The sensing scheme uses a wavelength interrogation method and the refractive index of a liquid is sensed by measuring the position of the dip in the reflected spectral intensity distribution. As an example, the aqueous solutions of ethanol with refractive indices in a range from 1.333 to 1.363 are measured. In addition, the increase in the sensitivity of the SPR fibre-optic refractive index sensor with the decrease of the fibre sensing length is demonstrated. [DOI: http://dx.doi.org/10.2971/jeos.2014.14033]
\end{abstract}

Keywords: Surface plasmon resonance, fibre-optic sensor, spectral interrogation technique, aqueous solutions of ethanol, refractive index

\section{INTRODUCTION}

The concept of optical sensing of various physical quantities based on surface plasmon resonance (SPR) phenomenon [1]-[3] has been proved to be very useful over the last several decades. Surface plasmons are charge density oscillations of conduction electrons - surface plasma waves - coupled to electromagnetic excitations propagating along the boundary between metal and dielectric material. Such waves are basically transverse magnetically polarized surface waves so that they can be resonantly excited only by a $p$-polarized incident light wave. The field of plasmons decays exponentially in the direction perpendicular to the boundary [4], in the metal as well in the dielectric material. When a specific resonance condition is fulfilled, maximum power of the incident light wave is coupled to the surface plasmons and the power carried by transmitted light wave drops down. Consequently, a sharp minimum (dip) is observed in the transmission spectrum. The resonance condition is highly sensitive to variations in the refractive index of surrounding medium (analyte). This mechanism gives the possibility to sense the changes of the refractive index of the analyte (usually liquid) via the shift of the dip in the transmission spectrum.

Because the excitation of surface plasmons by a light wave incident directly from air is not possible, the early sensing set-ups were based on the use of optical prisms. As an example, using the Kretschmann configuration, the base of a prism of high refractive index is coated with a thin metal film and the surface plasmons are excited in the metallic film by attenuated total reflection (ATR) mechanisms. The prism itself played the role of a coupling element between the evanescent wave excited on its base and the surface plasmons under the condition that the light beam incident angle exceeds its critical value. The evanescent wave passes through the thin metal film and the surface plasmons are excited at film lower boundary where the metal is in contact with the investigated analyte. SPR sensing can be performed in angular or spectral domain (angular or wavelength interrogation). In the angular interrogation, a monochromatic beam is used and the resonance angle shift is observed. In the wavelength interrogation, the resonance wavelength shift is observed.

The devices based on prism coupling exhibit high sensitivity and accuracy, but they are rather bulky and expensive, so they are mostly used in laboratory conditions. Naturally, optical fibre technology can be used to overcome these disadvantages and push the SPR sensing technology toward the industrial conditions. Sharing the same coupling mechanism based on the ATR, the prism coupler can be replaced by a bare optical fibre core covered by a thin metal layer. Because it is not possible to perform the detection in angular domain as in the case of prism-based devices, the SPR fibre-optic sensors are operated in a spectral domain observing the resonance wavelength shift. 
The fibre-optic sensors find a wide range of applications due to their simple structure, compact size, high accuracy and sensitivity. A large number of theoretical and experimental sensing schemes using a metal layer deposited on a bare core of an optical fibre have been reported [5]-[13]. In transmissionbased implementations of fibre-optic SPR sensors [5, 9], [10]-[13], the cladding/buffer layers are removed from the middle portion of the fibre and on the bare core is deposited a thin metal film, which is further surrounded by the analyte. Light from a white-light source is launched into one end of the fibre while the SPR spectrum is recorded at the other end of the fibre. The analyte is sensed via the shift of the dip in the transmission spectrum. In reflection-based implementations of fibre-optic SPR sensing schemes [5]-[8], the sensor utilizes a microfabricated mirror at the end of the fibre probe to reflect the light back through the fibre.

The first implementations of the SPR sensing structures were based on silver or gold layers mostly [5]-[13], because these metals exhibit sufficiently high free-electron density essential for effective excitation of surface plasmons. Although the structure based on a silver film is more sensitive, gold is still in favour because it offers better chemical stability. The performance of the sensor using other metals can be influenced by an unwanted oxide layer formed at the boundary exposed to the atmosphere. In order to avoid such degradation, the metal layer has to be protected by some overlayer [14], which can also be subject of naturally induced oxidation [15]. In addition, various attempts have been made to improve the sensitivity of the fibre-optic SPR sensors [7, 16, 17] and novel configurations with side-polished [18] or tapered optical fibres $[19,20]$ have been proposed. More recently, fibre-optic sensor implementations with thin films of the SPR supporting layers deposited by sputtering [21] were considered.

In this paper, a reflection-based fibre-optic refractive index sensor using an SPR in a thin metal film sputtered on a bare core of a multimode optical fibre is presented. The sensing element of the SPR fibre-optic sensor consists of a fused silica core of a step-index optical fibre with a gold film doublesided sputtered on the whole core surface. Thus, the thin film deposited also on the core end face of the fibre enables a reflection-based sensing scheme to be employed in measurement of the refractive indices of liquids. The sensing scheme uses a wavelength interrogation method and the refractive index of a liquid is sensed by measuring the position of the dip in the reflected spectral intensity distribution. The SPR fibre-optic sensor has been used to measure the aqueous solutions of ethanol with refractive indices in a range from 1.333 to 1.363 . In addition, the increase in the sensitivity of the SPR fibre-optic refractive index sensor with the decrease of the fibre SPR sensing length is demonstrated.

\section{EXPERIMENTAL}

\subsection{Fibre-optic SPR sensor}

For the fabrication of the fibre-optic SPR sensor, a polymerclad-silica (PCS) multimode optical fibre (QSF A 200, Quartz Products Corp.) with a core diameter of $200 \mu \mathrm{m}$ and numer-

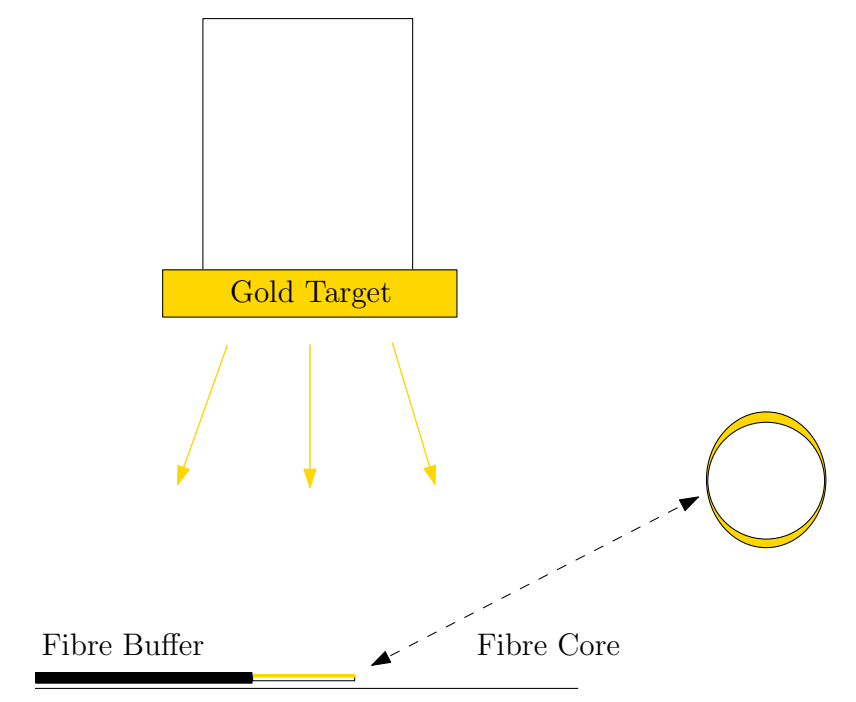

FIG. 1 Schematic illustration of a gold film deposition by magnetron sputtering on a fibre core.

ical aperture of 0.22 was used. The sensing probe was fabricated by first mechanically removing $12 \mathrm{~mm}$ in length of the buffer and cladding layers of the fibre. The resultant sensing area was then chemically cleaned.

The gold thin film on the fused-silica fibre core was prepared by radio-frequency magnetron sputtering (see Figure 1). A commercially available sputtering device (Leybold-Heraeus Z 550) was used with a partial pressure of argon of $150 \mathrm{mPa}$, a high frequency power of the magnetron of $50 \mathrm{~W}$ and a frequency of $13.56 \mathrm{MHz}$. The sputtering from a high purity (99.95\%) gold target was performed and the duration of deposition was so long to reach a $50 \mathrm{~nm}$ thickness. Thickness of the deposited gold film was measured on a glass plate (trial sample) by using an optical profilometer (Taylor Hobson, Ltd). A double-sided deposition technique was used to prepare the SPR layer as shown in Figure 1. Because of the geometry of the sputtering system, a gold layer of approximately $100 \mathrm{~nm}$ thickness was deposited directly onto the fibre end face to realize a terminated reflection-based fibre-optic SPR probe. The layer on the fibre end face, which acts as a mirror, is of sufficient thickness to provide adequate reflectivity, and not to support SPR at the end of the fibre. The sensing area of the probe is defined by an SPR supporting metal layer in contact with the optical fibre core free from the surrounding cladding/buffer layer.

\subsection{Test analyte}

As a test analyte, aqueous solutions of ethanol were prepared. The concentrations of the ethanol solutions were 0 (distilled water), 1.25, 2.5, 5, 10, 20, 30, 40, 50, 60 and 80 weight percent (wt.\%). The refractive indices of the solutions were independently measured at a wavelength of $589 \mathrm{~nm}$ by a digital refractometer (AR200, Reichert). The corresponding refractive indices of 1.3329, 1.3335, 1.3343, 1.3359, 1.3390, 1.3454, 1.3515, $1.3563,1.3594,1.3617$ and 1.3634 , respectively, were obtained at a temperature of $24.5^{\circ} \mathrm{C}$. 


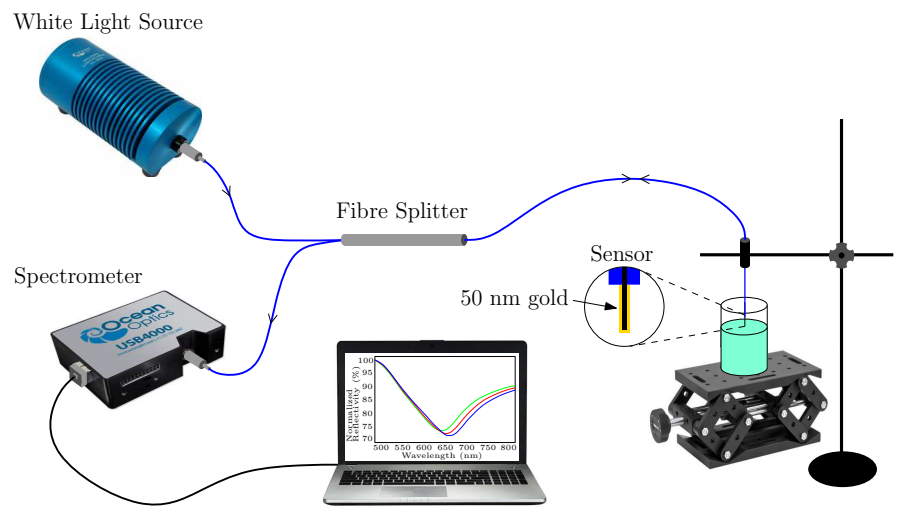

FIG. 2 Experimental set-up.

\subsection{Experimental set-up}

The experimental set-up used in measurements of SPR reflection spectra is shown in Figure 2. Light from a whitelight source, a halogen lamp (HL2000, Ocean Optics, Inc.), is launched into a multimode fibre splitter (SPLIT200-VIS-NIR, Ocean Optics, Inc.). One arm of the fibre splitter is connected to a fibre-optic probe with the terminated sensing part $12 \mathrm{~mm}$ long. The broadband light is transmitted through the probe to the sensing part where it is reflected by the fibre end face covered by the gold layer and goes back up the fibre-optic probe to the fibre splitter. The output arm of the fibre splitter is connected to a compact fibre-optic spectrometer (USB4000, Ocean Optics, Inc.) to measure an SPR reflection spectrum in a wavelength range from $400 \mathrm{~nm}$ to $1000 \mathrm{~nm}$.

\subsection{Experimental method}

All the SPR reflection spectra of the terminated SPR fibre-optic sensor were measured for the test analyte and were normalized by a reference spectrum. This was an air spectrum, since there was no SPR excitation in the considered wavelength range (400-1000 nm). A correct normalized SPR spectrum was measured by the spectrometer employing the relation

$$
R_{a n l}(\lambda)=\frac{I_{a n l}(\lambda)-I_{b k g}(\lambda)}{I_{a i r}(\lambda)-I_{b k g}(\lambda)},
$$

where $I_{a n l}(\lambda), I_{\text {air }}(\lambda)$ and $I_{b k g}(\lambda)$ are a test analyte spectrum, an air spectrum and a background spectrum, respectively. To find a resonance wavelength corresponding to minimum in the reflection spectrum (dip), smoothing with 50 computing points was performed on each spectrum to reduce the highfrequency noise.

\section{EXPERIMENTAL RESULTS AND DISCUSSION}

First, the reflection spectra of the SPR fibre-optic sensor probe were measured for the whole length $(12 \mathrm{~mm})$ of the fibre sensor exposed to the aqueous solutions of ethanol with refractive indices ranging from 1.3329 to 1.3634 . Figure 3 shows the measured normalized reflection spectra with varying refractive index of the analyte. It is clearly seen that the reflection spectrum corresponding to a particular refractive index of the analyte has a sufficiently pronounced dip. Thus, the end face

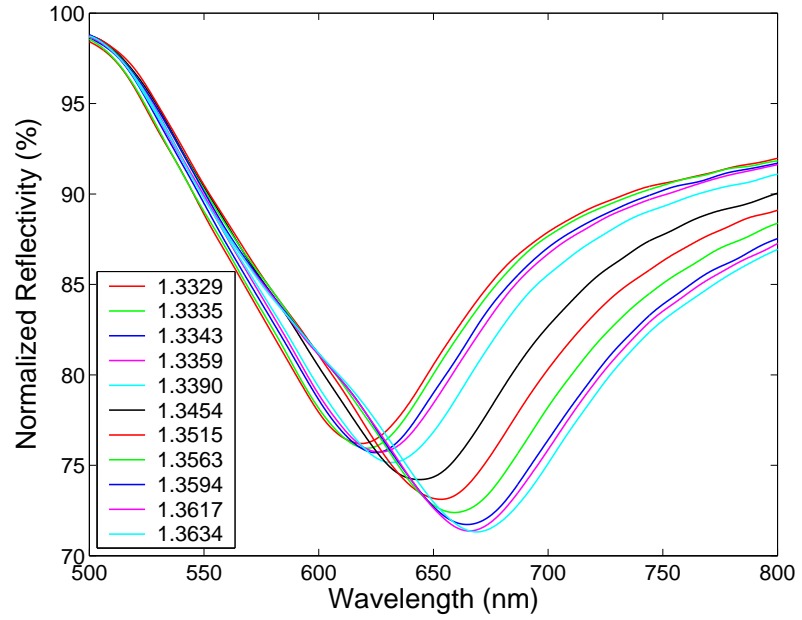

FIG. 3 SPR reflection spectra obtained after immersing the sensitive region of a $12 \mathrm{~mm}$ length in the aqueous solutions of ethanol.

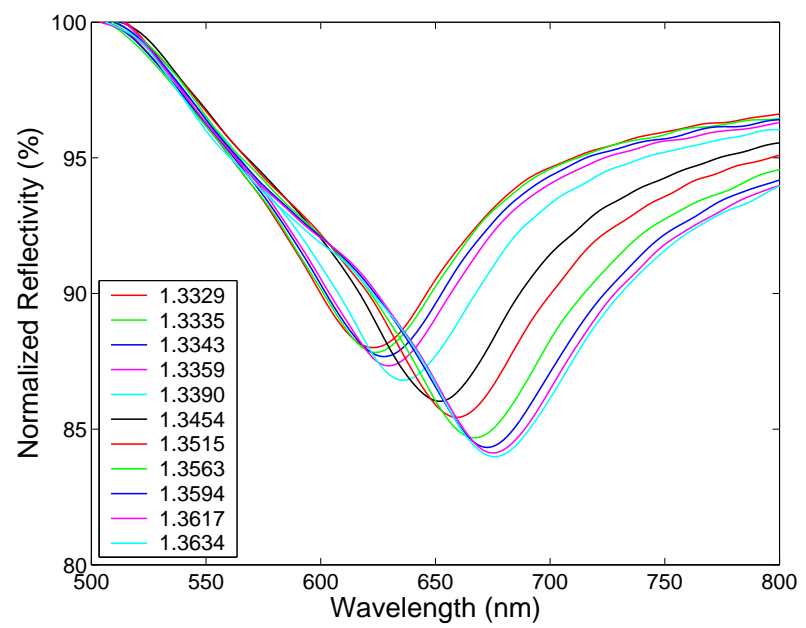

FIG. 4 SPR reflection spectra obtained after immersing the sensitive region of a $5 \mathrm{~mm}$ length in the aqueous solutions of ethanol.

mirror layer is of sufficient optical quality for reflection-based measurements. The dip is with a deeper resonance for a higher refractive index and the wavelength of the dip, the resonance wavelength, shifts to longer wavelengths with increasing the refractive index of the analyte.

One of the advantages of our arrangement of the SPR fibreoptic sensor is the possibility to vary the length of the fibre probe immersed in the analyte. A mini laboratory jack was used to lift the cell with the analyte so that the length of the fibre probe immersed in the analyte can be changed (see Figure 2). To reach a constant length of the immersed part of the probe during measurements, the level of the aqueous solution in the cell was inspected optically.

Next, the SPR reflection spectra were measured for a 9 and $5 \mathrm{~mm}$ immersed length. As an example, Figure 4 shows the normalized reflectivity spectra measured for the $5 \mathrm{~mm}$ long fibre sensor exposed to the aqueous solutions of ethanol. Once again, the SPR reflection spectra have sufficiently pronounced dips with a deeper resonance for the higher refractive index.

To compare the effect of the different sensing lengths of the 


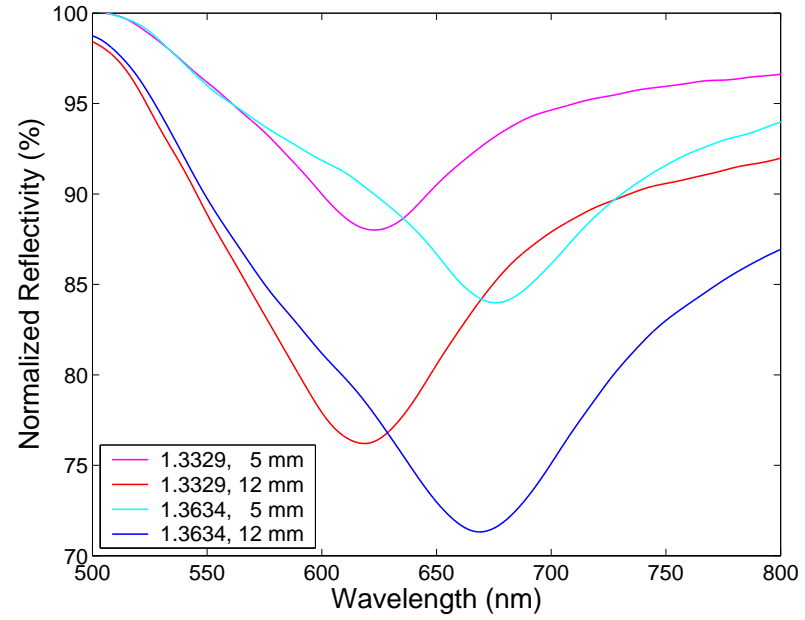

FIG. 5 Comparison of the SPR reflection spectra for two lengths of the sensitive region of the fibre.

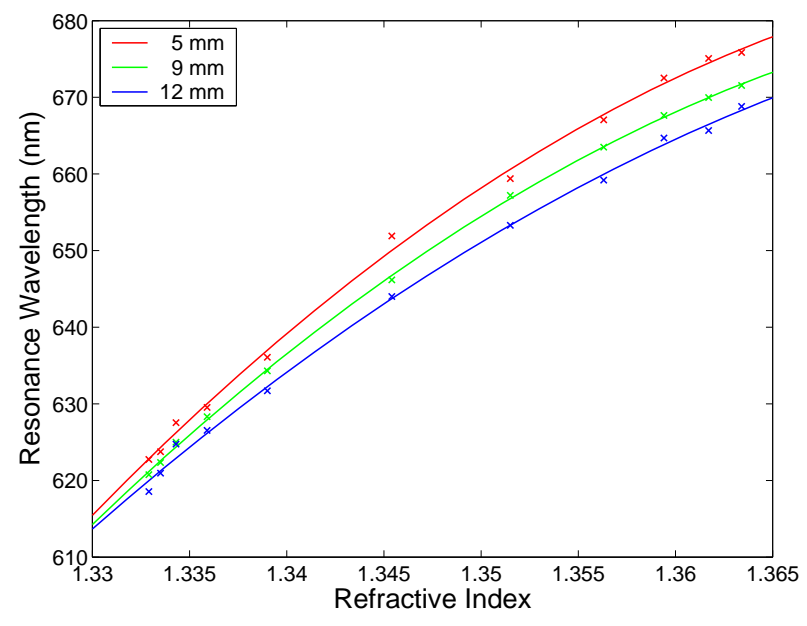

FIG. 6 The resonance wavelength as a function of the refractive index of the aqueous solution of ethanol for three lengths of the sensitive region of the fibre. The solid lines are polynomial fits.

SPR fibre probe on the measurement results, the SPR reflection spectra for the lowest and highest refractive index of the analyte are plotted in Figure 5 when the sensing length of the fibre is 12 or $5 \mathrm{~mm}$. The longer sensing length gives rise to the spectrum exhibiting a deeper resonance. This is due to increasing number of reflections taking place in the longer sensing part of the fibre. Moreover, the response characteristic is wider for the longer sensing length and the resonance wavelength is shifted to shorter wavelengths.

This shift is also illustrated in Figure 6, showing the measured resonance wavelength as a function of the refractive index of the analyte for all three sensing lengths of the fibre probe. The corresponding fitting functions (second-order polynomials) are shown in the same figure by solid lines. It is clearly seen that the resonance wavelength is with a greater shift for a lower refractive index. Similar behaviour, which differs from a steep increase of the resonance wavelength at higher refractive indices [6], was revealed for a transmission-based SPR sensor with a double-layer uniform-waist tapered fibre [22].

The non-linear response in Figure 6 indicates that the sensitiv-

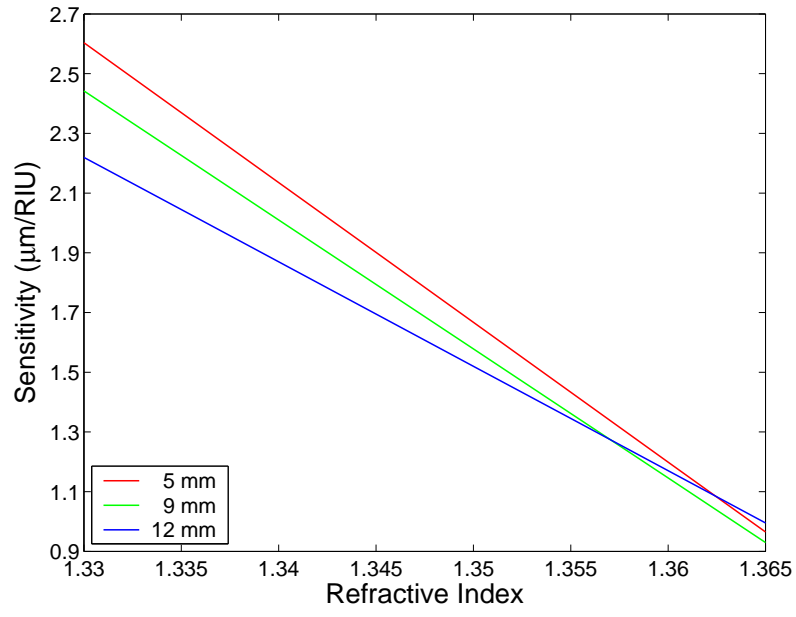

FIG. 7 The sensitivity as a function of the refractive index of the aqueous solution of ethanol for three lengths of the sensitive region of the fibre.

ity $S$, defined as the change of the position of the dip $\delta \lambda_{\mathrm{D}}$ with respect to the change of the refractive index $\delta n$ of the analyte, expressed in refractive index units (RIU), is RIU-dependent, with a higher sensitivity for lower RIUs. The corresponding sensitivity $S$ of the fibre-optic SPR sensor to the refractive index is evaluated by using equation

$$
S=\frac{\delta \lambda_{\mathrm{D}}}{\delta n}
$$

and it is plotted in Figure 7. The sensitivity is decreasing with RIU and reaches a higher sensitivity at lower RIUs. This dependence differs from increased sensitivity at higher RIUs for SPR sensors with a fully symmetric metal layer deposited on the fibre core [5]-[7]. The shorter the sensing length of the fibre probe the higher the sensitivity and for $12 \mathrm{~mm}$ length of the sensing fibre it reaches $1433 \mathrm{~nm} /$ RIU at 1.3549 RIU.

\section{CONCLUSIONS}

In this paper, a reflection-based fibre-optic refractive index sensor using SPR in a thin metal film deposited by sputtering on a bare core of a PCS multimode fibre has been presented. The sensing element of the SPR fibre-optic sensor is a fused silica core of a step-index optical fibre with a double-sided deposited gold film. The gold film is deposited not only on the core surface but also onto the core end face so that a reflectionbased sensing scheme to measure the refractive indices of liquids can be utilized. A wavelength interrogation method is used and the refractive index of a liquid is sensed by measuring the position of the dip in the reflected spectral intensity distribution. The sensing scheme has been applied to measure the refractive index of the aqueous solutions of ethanol in a range from 1.333 to 1.363 corresponding to the concentration in a range from 0 to $80 \mathrm{wt} . \%$. In addition, it is demonstrated that when the fibre SPR sensing length decreases, the sensitivity of the SPR fibre-optic refractive index sensor increases.

The main advantages of the realized fibre-optic SPR refractive index sensor include a reflection-based sensing scheme and possibility to change the sensitivity in a simple way. Moreover, the end face mirror layer required for reflection-based sensor 
can be deposited together with the SPR layer in one simple process (magnetron sputtering) without the need of more sophisticated techniques such as thermal evaporation, electron beam evaporation, etc.

\section{ACKN OWLEDGEMENTS}

The research was partially supported by the COST TD1001 action "OFSeSa" through project LD12003.

\section{References}

[1] E. Kretschmann, and H. Raether, "Radiative decay of nonradiative surface plasmons excited by light," Z. Naturforschung A23, 2135-2136 (1968).

[2] A. Otto, "Excitation of nonradiative surface plasma waves in silver by the method of frustrated total reflection," Z. Phys. 216, 398-410 (1968).

[3] H. Raether, Surface Plasmons on Smooth and Rough Surfaces and on Gratings (Springer-Verlag, New York, 1988).

[4] J. M. Pitarke, V. M. Silkin, E. V. Chulkov, and P. M. Echenique, "Theory of surface plasmons and surface-plasmon polaritons," Rep. Prog. Phys. 70, 1-87 (2007).

[5] R. C. Jorgenson, and S. S. Yee, "A fiber-optic chemical sensor based on surface plasmon resonance," Sensor. Actuator. A-Phys. 12, 213-220 (1993).

[6] R. C. Jorgenson, and S. S. Yee, "Control of the dynamic range and sensitivity of a surface plasmon resonance based fiber optic sensor," Sensor. Actuator. A-Phys. 43, 44-48 (1994).

[7] H. Suzuki, M. Sugimoto, Y. Matsui, and J. Kondoh, "Effects of gold film thickness on spectrum profile and sensitivity of a multimodeoptical-fiber SPR sensor," Sensor. Actuator. B-Chem. 132, 26-33 (2008).

[8] Y. Zhao, Z.-Q. Deng, and Q. Wang, "Fiber optic SPR sensor for liquid concentration measurement," Sensor. Actuator. B-Chem. 192, 229-233 (2014).

[9] R. Slavík, J. Homola, J. Čtyroký, and E. Brynda, “Novel spectral fiber optic sensor based on surface plasmon resonance," Sensor. Actuator. B-Chem. 74, 106-111 (2001).

[10] M. Mitsushio, K. Miyashita, and M. Higo, "Sensor properties and surface characterization of the metal-deposited SPR optical fiber sensors with $\mathrm{Au}, \mathrm{Ag}, \mathrm{Cu}$, and $\mathrm{Al}$," Sensor. Actuator. A-Phys. 125, 296-303 (2006).
[11] N. Rajan, S. Chand, and B. D. Gupta, "Fabrication and characterization of a surface plasmon resonance based fiber-optic sensor for bittering component," Sensor. Actuator. B-Chem. 115, 344-348 (2006).

[12] N. Rajan, S. Chand, and B. D. Cupta, "Surface plasmon resonance based fiber-optic sensor for the detection of pesticide," Sensor. Actuator. B-Chem. 123, 661-666 (2007).

[13] S. K. Srivastava, R. Verma, and B. D. Cupta, "Surface plasmon resonance based fiber optic sensor for the detection of low water content in ethanol," Sensor. Actuator. B-Chem. 153, 194-198 (2011).

[14] P. Bhatia, and B. Gupta, "Surface-plasmon resonance-based fiberoptic refractive index sensor: sensitivity enhancement," Appl. Optics 50, 2032-2036 (2011).

[15] D. Ciprian, and P. Hlubina, "Theoretical model of the influence of oxide overlayer thickness on the performance of a surface plasmon fibre-optic sensor," Meas. Sci. Technol. 24, 025105 (2013).

[16] A. Hanning, J. Roeraade, J. J. Delrow, and R. C. Jorgenson, “Enhanced sensitivity of wavelength modulated surface plasmon resonance devices using dispersion from a dye solution," Sensor. Actuator. B-Chem. 54, 25-36 (1999).

[17] R. K. Verma, and B. D. Gupta, "Theoretical modeling of a bidimensional U-shaped surface plasmon resonance based fiber optic sensor for sensitivity enhancement," J. Phys. D Appl. Phys. 41, 095106 (2008).

[18] H.-Y. Lin, W.-H. Tsai, Y.-C. Tsao, and B.-C. Sheu, "Side-polished multimode fiber biosensor based on surface plasmon resonance with halogen light," Appl. Optics 46, 800-806 (2007).

[19] F. J. Bueno, Ó. Esteban, N. Díaz-Herrera, M.-C. Navarrete, and A. Conzález-Cano, "Sensing properties of asymmetric doublelayer covered tapered fibers," Appl. Optics 43, 1615-1620 (2004).

[20] Ó. Esteban, N. Díaz-Herrera, M.-C. Navarrete, and A. GonzálezCano, "Surface plasmon resonance sensors based on uniformwaist tapered fibers in a reflective configuration," Appl. Optics 45, 7294-7298 (2006).

[21] S. Lopez, I. del Villar, C. Ruiz Zamarreño, M. Hernaez, F. J. Arregui, and I. R. Matias, "Optical fiber refractometers based on indium tin oxide coatings fabricated by sputtering," Opt. Lett. 37, 28-30 (2012).

[22] M.-C. Navarrete, N. Díaz-Herrera, A. González-Cano, and Ó. Esteban, "A polarization-independent SPR fiber sensor," Plasmonics 5 , 7-12 (2010). 